\title{
Vom processual shift zum complexity shift: Aktuelle analytische Trends der Grenzforschung
}

\author{
Christian Wille
}

\begin{abstract}
Die Auffassung, dass Grenzen aus sozialer Praxis hervorgehen und soziale Praxis hervorbringen, hat in den letzten Jahrzehnten für eine Reihe an Ansätzen und Begriffen gesorgt. Der Beitrag versucht, diese zu systematisieren, und stellt einen processual shift, multiplicity shift und complexity shift vor. Diese Trends beschreiben verschiedene analytische Schwerpunktsetzungen und eine zunehmend differenziertere Beschäftigung mit Grenzen. Abschließend wird auf die noch unzureichend bestimmte analytische Kategorie der sozialen Praxis und auf das Potential der Praxistheorien für die Grenzforschung eingegangen.
\end{abstract}

\section{Schlagwörter}

Bordering, borderwork, bordertextures, Multiplizität, Komplexität

\section{Einleitung}

Die Grenzforschung zählt seit den letzten Jahrzehnten zu den aufstrebenden Arbeitsfeldern der Sozial- und Kulturwissenschaften. Neben theoretisch-konzeptionellen Wenden (turns) ist dies vor allem auf jüngste gesellschaftliche Entwicklungen und das in Politik und Wissenschaft verstärkt nachgefragte Wissen über Grenz- und Migrationsdynamiken zurückzuführen. In diesem Zuge erfährt die Grenzforschung nun auch in Europa eine fortschreitende Institutionalisierung (Forschungszentren/-schwerpunkte, Studiengänge/-module, Handbücher u.a.), ebenso wie sie zunehmend mehr Disziplinen einschließt und sich in verschiedene Orientierungen ausdifferenziert (Comparative Border Studies, Critical Border Studies, Cultural Border Studies u.a.). Diese Entwicklung spiegelt sich gleichermaßen in den verwendeten Ansätzen und Begrifflichkeiten wider, die verschiedenen Disziplinen, Wissenschaftskulturen und Sprachgemeinschaften entspringen. Die Heterogenität und mitunter Schnelllebigkeit des Analyse- und Begriffsinstrumentariums erschwert die disziplinenübergreifende Selbstverständigung innerhalb der Grenzforschung, allerdings setzt sie zugleich Impulse für wissenschaftliche Weiterentwicklungen. Solchen wendet sich dieser Beitrag zu und versucht ungeachtet der vielfältigen Erkenntnisinteressen, Ansätze und Begriffe zentrale theoretisch-konzeptionelle Entwicklungen der letzten Jahrzehnte herauszuarbeiten. Damit soll zugleich ein Beitrag für die stärkere Konzeptualisierung und Systematisierung der Grenzforschung geleistet werden, wie sie etwa im Rahmen der Europa-Konferenz der Association for Borderlands Studies „Differences and discontinuities in a ,Europe without borders"“ (04.-07.10.2016, Luxemburg) eingefordert wurden.

Als ein allgemeiner Trend in der Grenzforschung ist in den letzten Jahrzehnten die Thematisierung der Grenze als Praxis festzustellen, die eine Reihe an Ansätzen und Begriffen mit geteilten Grundannahmen hervorgebracht hat. Diese in eine Abfolge ihrer Vorkommen zu bringen, ist in dem unübersichtlichen multidisziplinären Arbeitsfeld kaum leistbar, findet die theoretisch-konzeptionelle Debatte hier doch noch augenfälliger als andernorts in unterschiedlichen Geschwindigkeiten und Intensitäten statt. Für das Anliegen einer Systematisierung allerdings soll dieser Umstand aufgelöst werden, indem idealtypisch drei analytische Trends 
unterschieden werden. Diese Trends werden im Folgenden als shifts bezeichnet und zeigen weder grundlegende Neuorientierungen innerhalb der Grenzforschung an noch stehen sie hierarchisch zueinander oder lösen in der Zeit einander ab. Sie stehen jeweils für eine spezifische Orientierung in der wissenschaftlichen Auseinandersetzung mit Grenzen, schließen an generelle sozialanalytische Entwicklungen an und werden in der aktuellen Grenzforschung nebeneinander und miteinander praktiziert.

Die vorzustellenden shifts sind zunächst auf eine gesteigerte Aufmerksamkeit (in Europa) für Grenzen zurückzuführen, die aus dem Spannungsfeld von ,grenzenloser Welt` und ,Festung Europa' der 1990er-Jahre resultiert. Damit angesprochen ist die Gleichzeitigkeit von Globalisierungsdynamiken und des Falls des Eisernen Vorhangs einerseits sowie der Entstehung neuer Nationalstaaten und der Stabilisierung der EU-Außengrenzen andererseits. Diese widersprüchlich erscheinenden Entwicklungen haben für den Gegenstand der Grenze in der Weise sensibilisiert, dass Sabine Hess (2018, S. 84) rückblickend von einem border turn spricht und sich in der Grenzforschung eine tiefgründige Neuorientierung vollzog, welche die Idee der gesetzten und fixen Grenzen überwindet. Diese Neuorientierung bildet den Ausgangspunkt der vorzustellenden shifts und geht auf den von Theodore Schatzki, Karin Knorr Cetina und Eike von Savigny (2001) ausgerufenen practice turn zurück. Als solcher wird die Hinwendung zu einem Verständnis von Kultur bzw. Sozietät bezeichnet, das weniger von den Repräsentationen (Sprache, Zeichen, semiotische Strukturen) her gedacht wird, denn vielmehr durch die sich vollziehenden Praktiken bzw. Praktikenkomplexe (vgl. Reckwitz 2003). Die Praxisperspektive betont das performative Moment - etwa Akte der Einsetzung oder Anfechtung von Grenzen als Grenz(re)produktionen - unter Einbezug des darin wirksamen Wissens, der Diskurse, Tätigkeiten, Objekte, Körper und ihrer Settings. Die folgenden shifts knüpfen an dieses Verständnis an und stellen die soziale Praxis mit jeweils unterschiedlicher Akzentuierung in den Mittelpunkt. Die Schlussbetrachtung in diesem Beitrag wird allerdings zeigen, dass in der Grenzforschung eine noch weitgehend unbestimmte Verwendung des Praxisbegriffs auszumachen ist.

\section{Processual shift}

Die Rezeption des practice turn (vgl. Schatzki et al. 2001) lässt sich in der Grenzforschung zunächst an einem Grenzverständnis festmachen, das auch als konstruktivistisch bezeichnet wird (vgl. z.B. Bürkner 2017; Herzog/Sohn 2019). Es überwindet die Vorstellung der fixen und gesetzten Grenzen zugunsten der Auffassung, dass Grenzen die Ergebnisse von sozialen Prozessen seien (vgl. z.B. Newman/Paasi 1998; zur Übersicht Konrad 2015). Dieser einschneidende Perspektivwechsel ist zum einen auf die angedeutete Gegenerzählung zum Globalisierungsdiskurs zurückzuführen, zum anderen auf den sich seit den 1980er-Jahren vollziehenden spatial turn (vgl. z.B. Soja 1989; Lefebvre 1991), der die in den 1990er-Jahren noch stark nationalräumlich orientierte Grenzforschung nachhaltig beeinflusste. Denn so wie Raum oder Territorium nun nicht mehr als unhinterfragte Randbedingung des Sozialen, sondern als soziale Produktionen thematisiert werden, scheinen unter sogenannten postmodernen Bedingungen auch Grenzen adäquater als soziale Produktion beschreibbar und verstehbar zu werden. Mit dieser Betrachtungsweise vollzieht sich in der Grenzforschung eine Verschiebung des Blicks von der Grenze hin zu den Prozessen, die Grenzen hervorbringen. Diese Neueinstellung der Analyse zielt damit nicht länger auf die Grenze als ontologischen Gegenstand, sondern auf 
Prozesse der (De-)Stabilisierung von Grenzen, wie sie sich etwa über Praktiken vollziehen (vgl. Newman 2006; Kaiser 2012).

Die Prozessperspektive auf Grenzen ist nicht nur dem Anliegen geschuldet, gesellschaftlichräumliche Verhältnisse adäquater untersuchen zu können, sie erlaubt auch dafür einen theoretischen Rahmen zu formulieren, der folgendermaßen zusammengefasst werden kann:

- Gemachtheit von Grenzen: Grenzen werden nicht als fixe und gesetzte Gebilde betrachtet, sondern als Ergebnisse von sozialen Prozessen der Grenz(de)stabilisierung. Solche Prozesse können unterschiedlicher Art sein und verschiedene Maßstabsebenen umfassen, ebenso wie die daraus resultierenden Materialisierungen und Wirkungsweisen. Damit angesprochen sind beispielsweise im Kontext von Migration Praktiken des Protests, der Solidarität, der Kontrolle oder der Gesetzgebung, die sich auf unterschiedliche Weisen artikulieren.

- Fortwährende (Re-)Produktion von Grenzen: Außerhalb sozialer Prozesse sind Grenzen nicht denkbar, erst in und durch soziale Prozesse erlangen sie eine Relevanz und damit ihre Existenz. Grenzen müssen dementsprechend Gegenstand sozialer Auseinandersetzung sein, um als solche zu existieren. Insofern sind auf Dauer gestellte Grenzen stets als (Re-)Produktionen zu verstehen, die sich über kontinuierliche Prozesse der Grenz(de)stabilisierung realisieren. Dazu zählen auch Praktiken der Grenzverletzung, welche die Grenze als solche bestätigen und (re)produzieren.

- Veränderbarkeit von Grenzen: Die fortwährende (Re-)Produktion von Grenzen impliziert Verschiebungen und räumt damit einen potentiellen Wandel der praktizierten Grenz(de)stabilisierungen ein. Die stets im Werden befindlichen Grenzen unterliegen somit der Dynamik ihrer (Re-)Produktionen, die allerdings nicht festgeschrieben, sondern als kontingent vorausgesetzt werden. Ferner impliziert die (Re-)Produktionsdynamik eine zeitliche Dimension, die neben ihrem ephemeren Charakter auf ein Davor verweist und damit auf die in Grenz(de)stabilisierungen wirksame Gewordenheit von Grenzen.

- Ordnungen der Grenze: Grenz(de)stabilisierungen (re)produzieren Ordnungen, die sich in unterschiedlicher Weise materialisieren; so z.B. territoriale oder kategoriale Ordnungen, die variabel sind und Machtverhältnisse anzeigen, weshalb Grenz(de)stabilisierungen stets als politisch zu betrachten sind.

Diese vom Autor formulierten Punkte umreißen die Grundannahmen der Prozessperspektive auf Grenzen und verweisen auf eine Reihe an Erkenntnisinteressen, die im Zuge des processual shift verstärkt sichtbar werden und das Arbeitsfeld bis heute kennzeichnen. So interessieren wie unten zu sehen sein wird - im Hinblick auf Grenz(de)stabilisierungen etwa die beteiligten Akteur*innen, die eingelassenen sozialen Logiken, relevanten Maßstabsebenen, soziomateriellen Apparaturen ebenso wie Pfadabhängigkeiten oder Wandlungsprozesse in der Zeit sowie die sich in Grenz(de)stabilisierungen abzeichnenden Hegemonialverhältnisse.

Die Justierung und Erweiterung der Erkenntnisinteressen haben nicht nur dazu geführt, Grenzen und gesellschaftlich-räumliche Verhältnisse, die von gegenläufigen Entwicklungen und erhöhter Wandlungsdynamik geprägt sind, besser zu erfassen und zu verstehen. Sie haben auch eine Vervielfältigung der Disziplinen bewirkt, die Grenzforschung betreiben. Dabei hat sich disziplinenübergreifend ein Ansatz durchgesetzt, der die Vorstellung der Grenze als soziale Produktion eingängig auf den Begriff bringt und mit verschiedenen Grenzbegriffen (boundary, border, frontier, limit etc.) vereinbar ist. Angesprochen ist der Bordering-Ansatz, der die Grenze in prozessualisierender Absicht als Praxis fasst. Dieser Ansatz, der mitunter auch als doing border, border-making oder making difference umschrieben wird (vgl. z.B. Salter 2012), ist 
allerdings nicht als ein heuristisches Analyseinstrument zu verstehen, das appliziert wird und Grenzen unmittelbar als Ent- oder Vergrenzungen beschreibt und analysierbar macht. Bordering, so Yuval-Davis et al. (2019, S. 5), „[...] constitutes a principal organising mechanism in constructing, maintaining, and controlling social and political order". Es handelt sich also um einen Ansatz, der auf Grenz(de)stabilisierungen bzw. ihre Mechanismen fokussiert und in Orientierung an den jeweils spezifischen Erkenntnisinteressen und Untersuchungsgegenständen in ein adäquates Forschungsdesign übersetzt werden muss.

Die Etablierung des Bordering-Ansatzes im Sinne einer generellen Untersuchungseinstellung lässt sich weder eindeutig datieren noch einem*r bestimmten Autor*in zuordnen. Er wird schon Mitte der 1990er-Jahre in den Politikwissenschaften erwähnt (vgl. Albert/Brock 1996), zur Jahrtausendwende von Humangeograph*innen produktiv gemacht (s. unten; Houtum/Naerssen 2002), in anderen Disziplinen wird er erst später rezipiert. Die zeitlich versetzte Auseinandersetzung mit Bordering-Prozessen hat die Debatte über Grenzen fortlaufend stimuliert und begrifflich sowie konzeptionell weiter geschärft. So werden zum Beispiel Grenzpraktiken bzw. Praktiken der Grenze (z.B. Parker/Adler-Nissen 2012, S. 776; Wille et al. 2014, S. 10, 254ff.) - wie etwa das Zählen und Klassifizieren von Flüchtenden oder das diskursive Projizieren von Differenzen (othering) - als mögliche Operationalisierungen des Bordering-Ansatzes thematisiert (vgl. auch Lehner in diesem Band). Außerdem ist eine Unterscheidung zwischen De- und Rebordering-Prozessen auszumachen, über die das dynamische Zusammenspiel von Prozessen der Destabilisierung und Stabilisierung von Grenzen gefasst wird (vgl. z.B. Salter 2012; YuvalDavis et al. 2019, S. 59). So wie De- und Rebordering-Prozesse als einander unhintergehbar betrachtet werden, werden auch die in Grenzen codierten Ordnungen in eine sogenannte B/Ordering-Perspektive gestellt, die eine soziale und räumliche Dimension von Grenz(de)stabilisierungen analytisch unterscheidet. Dies zeigen exemplarisch Henk van Houtum, Olivier Kramsch und Wolfgang Zierhofer im Sammelband B/ordering Space (2005). Die Herausgeber veröffentlichen darin Beiträge von Humangeograph*innen, die sich mit Grenzpraktiken in einer sogenannten globalen und entgrenzten Welt auseinandersetzen und Bordering- mit Ordering-Prozessen in einer räumlichen Perspektive verknüpfen. Hintergrund dafür ist die Feststellung, dass sich durch Globalisierungsdynamiken nicht ,alles'verflüssigt und enträumlicht, sondern Praktiken stets Ordnungen und Abgrenzungen implizieren, die in räumlichen Differenzierungen manifest werden oder sich aus ihnen ergeben. Dieser Zusammenhang, den van Houtum und van Naerssen (2002, S. 134) auch mit „Making others through the territorial fixing of order“ umschreiben, beruht auf einem Verständnis von Grenze als „ongoing strategic effort to make difference in space“ (ebd., S. 126).

Der processual shift, der die Orientierung an Grenzen als ontologische Gebilde aufbricht, kann schließlich über zwei methodologische Prinzipien charakterisiert werden: Zum einen über die Dezentrierung der Grenze, womit nicht länger die Fokussierung auf die ,Mauer in der Landschaft', sondern die Fokussierung auf soziale Prozesse ihrer Einsetzung oder Anfechtung angesprochen ist. Zum anderen über die Prozessualisierung der Grenze, die dynamische Veränderung sowie historische Gewordenheit einräumt und die Grenze nur in ihren (Re-)Produktionen existieren lässt. Beide Prinzipien sind ausschlaggebend für die zunehmende Relativierung von nationalräumlichen Ordnungen als Deutungs- und Erklärungskategorien in der Grenzforschung zugunsten von Analyseaspekten, die territoriale und nichtterritoriale Grenzen als soziale (Re-)Produktionen bestimmbar machen. Die sich im ersten Jahrzehnt des 21. Jahrhun- 
derts durchsetzende Einsicht um die Vielfältigkeit der für solche (De-)Stabilisierungsprozesse relevanten Aspekte wird vom Autor im Folgenden als multiplicity shift bezeichnet.

\section{Multiplicity shift}

Die den multiplicity shift charakterisierenden Entwicklungen sind als Fortschreibung und Erweiterung des processual shift zu verstehen. Grenzen stehen hier weiter als soziale Produktionen im Zentrum, sie erfahren nun allerdings eine nähere Bestimmung. Sie beruht auf der Beobachtung, dass Grenz(de)stabilisierungen selten in binären Codierungen aufgehen, von nur einem*r Akteur*in mit eindeutiger Agenda und Identität ausgehen oder sich an einem bestimmten Ort in expliziter Weise materialisieren. Grenz(de)stabilisierungen sind deutlich vielfältiger angelegt, weshalb sie zunehmend als multiple Prozesse verstanden und als solche untersucht werden. Dafür kennzeichnend ist eine Annäherung an Grenzen aus unterschiedlichen Blickwinkeln, über die möglichst viele der relevanten Aspekte und Perspektiven auf Grenz(re)produktionen einbezogen werden (vgl. Rumford 2012). Dieses an Multiplizität interessierte Vorgehen nimmt die soziale Gemachtheit von Grenzen in ihren unterschiedlichen Facetten ernst und hat nicht zuletzt das Arbeitsfeld für kulturwissenschaftliche Zugänge weiter geöffnet.

Für die analytische Perspektivweitung im Zuge des multiplicity shift haben vor allem die Vertreter*innen der Critical Border Studies sensibilisiert, deren Anliegen machtkritisch orientiert sind (vgl. z.B. Parker et al. 2009; Parker/Vaughan-Williams 2012; Salter 2012; Brambilla/Jones 2019; Jones 2019; Yuval-Davis et al. 2019) und auf einer eingängigen Überlegung beruhen: „the construction of borders [...] must always be done somewhere by someone against some other" (Tyerman 2019, S. 2). Sie interessieren sich also nicht nur dafür, wie Grenzen (re-)produziert werden, sondern auch von wem (De-)Stabilisierungsprozesse mit welchen Interessen, Adressierungen und Effekten ausgehen. Anfang der 2010er-Jahre konstatieren sie vielschichtiger werdende bordering practices und zugleich ein dafür unzureichendes theoretisch-konzeptionelles Analyseinstrumentarium. Inspiriert von den dargelegten Entwicklungen des processual shift und mit Blick auf nationale Grenzen unterstreichen sie, dass sich Grenz(de)stabilisierungen nicht unmittelbar als solche zu erkennen geben und untersucht werden müsse, mit welchen Konsequenzen Grenz(de)stabilisierungen in Erscheinung treten und wer davon in welcher Weise profitiert. Bordering practices als Analysekategorien sollten daher weitgreifend im Sinne dynamischer Sets von performances bzw. Praktiken, die auch alltägliche Lebenswirklichkeiten einschließen und durch die Grenze gedacht werden, angelegt sein (Parker/Vaughan-Williams 2012, S. 728f.; vgl. auch Rumford 2011). Das zuletzt genannte Vorgehen, das Rumford (2012, S. 895) begrifflich als „seeing like a border“ fasst, bezeichnet das Anliegen der Grenze, in ihre sozialen Arenen zu folgen (vgl. auch Gerst/Krämer in diesem Band). Dorthin, wo die Grenze - in und durch Praktiken - als alltagskulturelle (Re-)Produktion stattfindet: „In aspiring to ,see like a border' we must recognise the constitutive nature of borders in social [...] life“ (Rumford 2012, S. 897). Die Vertreter"innen der Critical Border Studies machen sich somit für eine „multi-dimensional matrix of bordering“ (Konrad/Brunet-Jailly 2019, S. 5) stark, die differenzierend danach fragt, wie, wo und unter Wirksamkeit welcher Aspekte sich Grenz(de)stabilisierungen vollziehen: etwa in und durch Verkehrsinfrastrukturen, Kontrollprozeduren, Überwachungstechnologien, Repräsentation in Literatur und Kunst, Gesetzgebung, zivilgesellschaftliche Solidar- oder Protestkundgebungen, Symbole oder Rituale kultureller oder räumlicher Zugehörigkeit etc. Die Multiplizitätsperspektive erweitert somit das Spektrum der 
analytischen Ansatzpunkte und erlaubt Prozesse der Grenz(de)stabilisierung weitgreifender zu erfassen und damit einem simplifizierenden Verständnis von Grenzen entgegenzuwirken. Dies zeigen verschiedene Analyseperspektiven auf Grenz(de)stabilisierungen, die vor allem im Zuge des multiplicity shift diskutiert und in Anschlag gebracht werden:

- Akteurspluralität: In Prozessen der Grenz(de)stabilisierung wirken weder nur ein*e Akteur*in noch sind sie exklusiv an eine staatliche Autorität gekoppelt. An ihnen sind unterschiedliche Akteur*innen (zugleich) beteiligt, die Grenzen etwa durch Anfechtung, Subversion oder Rebordering (re)produzieren. Diese Analyseperspektive schließt staatliche Akteur*innen ebenso ein wie Nichtregierungsorganisationen (NGOs), Aktivist*innen, Kunstschaffende, den*die einzelne*n Bürger*in oder Schleuser*innen und Flüchtende. Ihre (potentielle) Partizipation an Grenz(de)stabilisierungen fasst Rumford (2012, S. 894) mit dem Begriff borderwork, der vor allem auf die Handlungsmacht von zivilgesellschaftlichen Akteur*innen verweist und ein politisches Kapital beschreibt, das Responsibilisierung und Intervention impliziert:

„Ordinary people (citizens, non-citizens) are increasingly active in constructing, shifting, or even erasing borders. Citizens, entrepreneurs, and ,civil society ' actors, amongst others, can engage in bordering, or what is here termed borderwork; the effort of ordinary people leading to the construction, dismantling, or shifting of borders. The borders concerned are not necessarily those (at the edges) of the nation-state; they can be found at a range of sites throughout society: in towns and cities, in local neighbourhoods, in the countryside" (ebd., S. 897).

- Diffundiertheit: Borderwork weist dem Zitat weiter folgend über (De-)Stabilisierungsprozesse hinaus, die sich an nationalen Rändern ereignen, und fasst Grenzen als diffundiert. Damit ist auf den Umstand verwiesen, dass Grenzen räumlich verstreut, vom tradierten Ort der Grenze entfernt und durchaus ,inmitten“ von Gesellschaften stattfinden. Diese Analyseperspektive folgt der These Balibars (1998) „borders are everywhere“ und begreift die Grenze als ein in vielfältigen Praktiken eingelassenes Phänomen, das sich an verschiedenen Orten (zugleich) und räumlich variabel artikuliert. Anschauliche Beispiele dafür geben rezente Externalisierungen und Internalisierungen, die sich in der Vervielfältigung und Verschiebung von Kontroll- und Regulierungspraktiken an Orte außer- und innerhalb von nationalen Territorien zeigen. Solche Diffundierungsprozesse steigern die Multiplizität von Grenz(de)stabilisierungen in räumlicher Hinsicht, zugleich erweitern sie das Spektrum der zu berücksichtigenden Akteur*innen, denn zumeist stehen Flüchtende oder ihre Migrationsdynamiken in Wechselwirkung mit Externalisierungs- und Internalisierungsprozessen; auch involviert das „territoriale Outsourcing“ von Kontrollpraktiken die Autoritäten weiterer Staaten, ebenso wie Grenzstabilisierungen im „nationalen Inneren“ etwa private (Sicherheits-)Dienste auf den Plan rufen (Risse 2018). Akteurspluralität und räumliche Diffundiertheit von Grenzen bilden auch die Fluchtpunkte für Anne-Laure Amilhat Szary und Frédéric Giraut (2015), die dem multiplicity shift folgend das Konzept der mobile borders vorlegen. Damit fassen sie begrifflich die Multilokalität und Dynamik von Grenzen und betonen, dass mobile Grenzen trotz der Relativierung des tradierten Orts der Grenze keineswegs als dematerialisiert aufzufassen sind, sondern sich ihre Materialisierungen vervielfältigt haben, in Bewegung und räumlich verstreut sind.

- Soziomaterialitäten: Das Konzept der mobile borders versucht die multiplen Erscheinungsformen von Grenzen zu erschließen und misst dafür dem Soziomateriellen einen besonderen 
Stellenwert bei. In den Blick geraten hier vor allem die an (De-)Stabilisierungspraktiken des Identifizierens, Kategorisierens, Filterns, Markierens, Steuerns usw. beteiligten technischen Apparaturen und ihre vielzähligen Bezüge zum menschlichen Körper. Ein bekanntes Beispiel dafür sind die in den USA und der Europäischen Union (EU) eingeführten smart borders (vgl. Sontowski 2018). Über biometrische Techniken, Datenüberwachung oder BigData-Automatisierung werden hier Körper und Apparaturen zu weiteren Akteur*innen von Grenz(de)stabilisierungen, indem sie soziomaterielle Allianzen eingehen und damit - wie Louise Amoore (2006, S. 347f.) es formuliert - Körper zu (mobilen) Trägern der Grenze werden (siehe auch Pötzsch in diesem Band). Solche Allianzen von Körpern und Überwachungstechnologien, die multiple Konfigurationen bilden können, thematisieren Amilhat Szary und Giraut (2015) über den Begriff borderity und bezeichnen damit das Verhältnis von Körper und Macht im Kontext der Grenze. (Biopolitische) Kontrolltechniken werden dabei als Praktiken der Grenzstabilisierung verstanden, mit denen Körper bzw. Subjekte wiederum auf unterschiedliche Weisen - etwa durch Destabilisierungen - in Beziehung stehen. Amilhat Szary und Giraut (2015) sprechen daher differenzierend von borderities (Plural), womit sie eine weitere Facette der Multiplizität von Grenz(de)stabilisierungen erschließen.

- Multivalenz: Der differenzierende Blick auf Soziomaterialitäten hilft zu bestimmen, inwiefern Körper in Grenz(de)stabilisierungsprozessen zu Akteur*innen werden bzw. Überwachungsapparaturen für bestimmte Körper wirksam werden. Dieses Wechselverhältnis ist jeweils variabel formuliert, da Grenz(de)stabilisierungen in Verbindung mit bestimmten Körpern bzw. Subjekten unterschiedliche Wertigkeiten entfalten. Diesen Zusammenhang umschreibt Marc B. Salter (2012, S. 750) in Bezug auf Balibar mit „the border is not everywhere for everyone" und verweist damit auf den multivalenten Charakter von Grenzen. Diesen thematisiert auch Julia Schulze Wessel (2017, S. 119ff.) mit Blick auf undokumentierte Migrant*innen und arbeitet angesichts ihrer andauernden Verflochtenheit mit Grenz(de)stabilisierungen für diese Gruppe eine „Permanenz der Grenze“ heraus. Sie besteht in der omnipräsenten Potentialität von Kontrollpraktiken, die sich weniger an nationalen Rändern denn vielmehr an strategisch wichtigen Punkten entlang bekannter Fluchtrouten (plötzlich) ereignen (können) (vgl. auch Vollmer/Düvell in diesem Band). Die Autorin bezeichnet undokumentierte Migrant"innen daher als „Bewohner der Grenze“, die sich fortwährend im „Raum der Grenze“ bzw. „in der Grenze“ bewegen (Schulze Wessel 2017, S. 123, Herv. i. O.). Dem stellt sie kontrastiv legal wandernde Personengruppen gegenüber und schlussfolgert, „[...] dass sich zwei Menschen an demselben Ort befinden können, ohne dass beide Teil der Grenze sein müssen“ (ebd., S. 134f.). Dieses Beispiel selektiver Wirksamkeit von Stabilisierungsprozessen untermauert die oben erwähnte Rolle des Körpers als (mobiler) Träger der Grenze, die Schulze Wessel (2016, S. 52) als ein Merkmal zeitgenössischer Grenzen herausstellt:

„[...] borders are no longer tied to places, but instead to persons, and this is probably the clearest difference between today's border and the traditional territorial boundary. Whereas nation-state borders enclosed a specific territory and were institutions designed to regulate cross-border movements and transactions, today's borders enclose certain persons.“

Diese und weitere Analyseperspektiven illustrieren den als multiplicity shift bezeichneten Trend, die für Grenzen konstitutiven Prozesse verstärkt in ihrer Vielschichtigkeit zu denken. 
Dafür werden in der Grenzforschung multiple Perspektiven auf Praktiken der Grenz(de)stabilisierung eingenommen, um möglichst vielfältige und zugleich jeweils spezifische Ansatzpunkte der Analyse herauszuarbeiten. Dieses Vorgehen ist von zwei methodologischen Prinzipien gekennzeichnet: Erstens von einer Multilokalisierung, über die der territoriale Rand als tradierter Ort der fixen Grenzen zugunsten der vielzähligen und wechselnden Orte, an denen sich Grenzen ereignen, relativiert wird. Solche Orte der Grenz(re)produktion können räumlich bestimmt werden, jedoch weist der multiplicity shift über die diffundierte Verräumlichung von Grenz(re)produktionen weit hinaus. Er macht vor allem auf die multiplen sozialen Schauplätze von Grenzen aufmerksam, an denen (De-)Stabilisierungen in unterschiedlicher Weise codiert sind und wirksam werden. Diese als analytische Ansatzpunkte herauszuarbeiten, gelingt, zweitens, über das Prinzip der Multidimensionierung, über das Prozesse der Grenz(de)stabilisierungen in verschiedene Analysedimensionen - etwa Akteurspluralität, Soziomaterialitäten, Multivalenz etc. - aufgefächert und in ihren vielfältigen Artikulationsformen zugänglich werden. Beide methodischen Prinzipien, die in einer weniger analytisch unterscheidenden Betrachtung auch als „multi-sited approach“ (Brambilla 2015, S. 22) bezeichnet werden, fördern nicht nur die Ansatzpunkte einer multiplen Analyse zu Tage, sie helfen auch das Modell der territorial-linienartig gedachten Grenze zu überwinden und sensibilisieren für Zusammenhänge zwischen den für Grenz(de)stabilisierungen relevanten Teilnehmer*innen und Dimensionen. Damit ist auf die in der Multiplizität angelegte Tendenz verwiesen, die sozialen (Re-)Produktionen von Grenzen in ihrer Komplexität und damit auch wissenschaftlich in komplexer Weise beschreiben und untersuchen zu wollen. Sie gewinnt in der Grenzforschung seit knapp einem Jahrzehnt an Bedeutung und deutet auf einen complexity shift hin.

\section{Complexity shift}

Als complexity shift wird in diesem Beitrag der Trend in der Grenzforschung zu komplexeren Betrachtungen bezeichnet. Dafür sind die oben erläuterten Entwicklungen weiterhin leitend, allerdings werden Grenz(de)stabilisierungen nunmehr weniger als ,überschaubare` Prozesse oder ausschließlich als Vielheiten der sie konstituierenden Analyseaspekte gefasst. Grenz(de)stabilisierungen werden hier als Effekte von dynamischen Formationen verstanden, die für performative Verweisungszusammenhänge zwischen Wissen, Diskursen, Tätigkeiten, Objekten, Körpern stehen und Grenzen hervorbringen bzw. durch Grenzen hervorgebracht werden. Dieser Zugang versucht die für Grenz(de)stabilisierungen wirksamen Elemente zu einer komplexen Formation zusammenzudenken und sie als sozial, materiell, räumlich und zeitlich bestimmbares Gefüge beschreibbar zu machen. Für eine solche Morphologie der Grenze werden Wissen, Diskurse, Tätigkeiten, Objekte, Körper als von bestimmten Beziehungen zusammengehalten vorausgesetzt und in ihrer situativen Aufeinanderbezogenheit betrachtet. Dabei wird weniger angestrebt, die solche Formationen konstituierenden Elemente erschöpfend zu erfassen, sondern spezifische Konstellationen zu identifizieren, die in und für Grenz(de)stabilisierungen wirksam sind. Dem schließen sich Fragen nach den Entwicklungspfaden solcher Formationen an, den darin eingelassenen Logiken oder nach der Handlungsmacht der darin verstrickten Subjekte; aber auch inwiefern sich Grenz(de)stabilisierungen durch die Wechselwirkungen von bestimmten Wissen, Diskursen, Tätigkeiten, Objekten und Körpern verstärken oder relativieren. Solche komplexeren Perspektivierungen von Grenzen sind zum Beispiel zunehmend bei der Untersuchung von Flüchtlingscamps zu beobachten, wenn diese als durch Grenzen hervorgebrachte dynamische Formationen und zugleich als Grenzen (re-)produzierende komplexe 
Gebilde projektiert werden. So betrachten etwa Diana Martin, Claudio Minca und Irit Katz (2019), Thom Tyerman (2019), Michel Agier (2018) oder Lucas Oesch (2017) Camps nicht als isolierte Einheiten, sondern als Praktikenkomplexe in und mit ihren Bezügen zu Grenzen: „Borders and bordering practices are [...] related to the appearance and the functioning of many contemporary camps" (Martin et al. 2019, S. 19). So wird etwa der Dschungel von Calais (vgl. Überblick bei Ibrahim/Howarth 2018) als „hypertrophie de la frontière“ (Agier 2018, S. 190) bezeichnet oder analytisch als „[a] complex assemblage of law, geography, technology, and policing [...]“ gefasst (Tyerman 2019, S. 7). In einer solchen analytischen Perspektive untersucht Tyerman (ebd.), wie in Calais Grenzen in und durch Praktiken (re-)produziert werden und rekonstruiert dort europäische Grenzen als lokal verleiblichte politische Wirklichkeiten:

„In Calais the ,grand' racially exclusionary geopolitics of European borders is enacted through ,minor' everyday practices, the interruption of meals, contamination of water, braking of tents, and physical assaults of migrants. [...] these everyday features of the border are nor incidental to its geopolitical power but rather give the ,hostile environment ${ }^{6}$ its texture, bringing an inequitable racialized global ontology intimately to life as an embodied political reality“ (ebd., S. 14).

Die Komplexitätsperspektive bringt weiter die im Zitat genannten Praktiken mit solchen der Grenzdestabilisierung in einen Zusammenhang: etwa mit Praktiken der Solidarität (z.B. Schweigemärsche), des Protests (z.B. Hungerstreik) oder der Subversion (z.B. Fluchtversuche). Grenzstabilisierungen und -destabilisierungen werden hier also als komplexe Formationen zusammengedacht, die sich in und durch „violent everyday [border] work“ (ebd.) fortlaufend (re-)strukturieren und „the ,grand" [...] geopolitics of European borders“ (ebd.) stabilisieren bzw. destabilisieren.

Die Ausführungen zu Calais zeigen, inwiefern die an Komplexität orientierte Grenzforschung synthetisch gedachte Einheiten adressiert, um über das relationale Zusammenwirken ihrer Konstituenten zu einem tieferen Verständnis von Grenz(de)stabilisierungen zu gelangen. Solche hier als Formationen umschriebene und am Beispiel von Flüchtlingscamps illustrierten Einheiten werden einschlägig auch als Ensemble, Komplexe, Assemblagen, Arrangements, Versammlungen oder auch als Regime, Netzwerke, Ecosysteme, Konglomerate, Konstellationen, textures oder scapes bezeichnet (vgl. z.B. Tsianos/Karakayali 2010; Casas-Cortes et al. 2015, S. 69f.; Brambilla et al. 2015; Sohn 2016; Gerst et al. 2018; Weier et al. 2018; Pott et al. 2018; Wille et al., i.E.). Einen besonderen Impuls für diese Betrachtungsweise von Grenzen hat der breit rezipierte Ansatz borderscapes gesetzt, der allerdings weder eine Theorie der Grenze noch eine abgezirkelte Heuristik bezeichnet. Borderscapes steht für eine kritische Forschungsperspektive auf Grenz(de)stabilisierungen, welche die Vielheit der daran beteiligten und räumlich sowie zeitlich verstreuten Akteur*innen, Praktiken und Diskurse berücksichtigt und ihr performatives Zusammenwirken als eine stets umkämpfte Formation versteht (vgl. Brambilla 2015; Brambilla/Jones 2019). Diese Konzeption, die in der aktuellen Grenzforschung unterschiedliche Auslegungen erfährt, vertritt auch der Ansatz bordertextures, der die für Grenz(de)stabilisierungen wirksamen Formationen von Wissen, Diskursen, Tätigkeiten, Objekten und Körpern als Texturen fasst. Der Texturbegriff soll die relationale Verflochtenheit solcher Konstituenten betonen, eine ,dichte Beschreibung' von Grenz(de)stabilisierungen befördern und in der Grenzforschung für die symbolisch-ästhetische Dimension und ihr politisches Potential sensibilisieren (vgl. Weier et al. 2018; Wille et al., i.E.). 
Beide Ansätze beziehen sich auf territoriale Grenzen und teilen eine für den complexity shift zentrale und bereits angedeutete Auffassung. Danach kennzeichnen sich Grenzen als Produkte und Produzent*innen von sozialer Praxis, die als soziomaterielle Formation verstanden allerdings näher bestimmbar ist, denn Ansätze wie borderscapes oder bordertextures unterstellen Formationen der Grenz(de)stabilisierung komplexe Struktur(ierung)en, die sich zum Beispiel in der Aushandlung von konkurrierenden Logiken zeigen, welche sich im relationalen Zusammenspiel von bestimmten Akteur*innen, Praktiken und Diskursen rekonstruieren lassen. Die Untersuchung solcher Formationen zielt damit stets auf einen Erkenntnisgewinn über die Infra-Struktur(ierung)en von Grenzen, die sich als borderscapes oder bordertextures materialisieren und als solche empirisch handhabbar werden. Die an Komplexität orientierte Grenzforschung stellt also auf die relationalen „Binnenstrukturen“ (Gerst et al. 2018, S. 5) von Grenzen $\mathrm{ab}$, die aus unterschiedlichen Blickwinkeln untersucht werden können und als Schauplätze der Aushandlung weder hierarchisch organisiert sind noch linear emergieren. Relationale Formationen der Grenz(de)stabilisierung werden als rhizomartig strukturiert verstanden, d.h. von vielzähligen Querverbindungen, Kreuzungen, Konnexionen etc. gekennzeichnet, die auf ein multiskalares und azentrisches Gefüge verweisen. Weiter unterliegen sie der Dynamik ihrer fortlaufenden (Re-)Produktion, weshalb sie nicht als fixe Formationen, sondern als volatil-veränderbare soziale Gefüge im Sinne eines vibrierenden Körpers der Grenze aufzufassen sind.

Solche komplexer angelegten Betrachtungen werden in der Grenzforschung zwar verstärkt eingefordert - wie etwa auf der Konferenz B/ORDERS IN MOTION: Current Challenges and Future Perspectives (15.-17.11.2018) in Frankfurt/Oder -, allerdings noch unzureichend realisiert. Dies kann mit dem noch relativ jungen Trend erklärt werden, aber auch mit dem ambitionierten Anspruch, die für Grenz(re)produktionen relevante Wissen, Diskurse, Tätigkeiten, Objekte und Körper nicht nur zu identifizieren und mehr oder weniger voneinander isoliert zu betrachten (multiplicity shift), sondern sie in ihrem performativen Zusammenwirken als dynamische Formation empirisch zu begreifen. Dafür können zwei methodologische Prinzipien in Anschlag gebracht werden, die helfen, Grenzen als komplexe Formationen und in ihrer Wirkmächtigkeit in den Blick zu bekommen. Dazu zählt das Texturieren, das im eigentlichen Wortsinn ein Verfahren der Textilindustrie bezeichnet, um glatten Garnen eine Kräuselstruktur zu verleihen und somit ihr Volumen zu verändern. Auch in der Grenzforschung kann das Texturieren als Technik dienen, um Texturen von Grenz(de)stabilisierungen - d.h. das darin wirksame Wissen, die Diskurse, Tätigkeiten, Objekte, Körper und ihre Verweisungszusammenhänge - aufzudecken bzw. sichtbar(er) zu machen. Texturieren als ein Gegen-den-Strich-Kämmen von Formationen der Grenz(de)stabilisierungen soll für invisibilisierte Konstituenten und implizite Verknüpfungen sensibilisieren und Hinweise auf mögliche analytische Zugriffspunkte geben, denn Grenzen als Formationen bieten keinen klar identifizierbaren Ausgangspunkt der Analyse; es kann lediglich an einigen zu Tage geförderten „Knotenpunkten` der Formation angesetzt und von hier aus entlang von Querverbindungen das soziomaterielle Gefüge weiter erschlossen werden. Solche Erschließungsprozesse werden durch das weitere Prinzip des Relationierens unterstützt, über das nicht nur die als relevant angenommenen Wissen, Diskurse, Tätigkeiten, Objekte und Körper suchend mit Blick auf etwaige Zusammenhänge zueinander in Beziehung gesetzt werden. Relationierungsprozesse orientieren sich vor allem an den fortlaufenden empirischen (Re-)Struktur(ierung)en solcher Formationen, für welche die erwähnten performativen Verweisungszusammenhänge virulent sind. Für das Auf- und Nachspüren solcher wechselseitigen Bezugnahmen spielen ethnografische und textanalytische 
Verfahren gleichermaßen eine Rolle, allerdings geben erst wenige Arbeiten Hinweise darauf, wie Komplexitäten und Performativitäten in der aktuellen Grenzforschung tatsächlich adäquat operationalisiert werden können.

\section{Kritische Schlussbetrachtung}

Die Grenzforschung ist zunehmend stärker herausgefordert im Hinblick auf Europäisierungsprozesse, Rebordering-Tendenzen, Migrationsdynamiken usw., was sich in einer bewegten theoretisch-konzeptionellen Debatte widerspiegelt. Sie hat in den letzten Jahrzehnten eine kaum überschaubare Reihe an Ansätzen und Begriffen hervorgebracht, die inspiriert vom practice turn (vgl. Schatzki et al. 2001) die Grenze als soziale Praxis in den Mittelpunkt stellt. Von dieser als übergreifende Verklammerung verstandenen Orientierung ausgehend wurden in diesem Beitrag drei analytische Trends der aktuellen Grenzforschung idealtypisch unterschieden. Die als shifts bezeichneten Trends standen dabei für spezifische Schwerpunktsetzungen in der Beschreibung und Analyse von Grenzen: So wurde zunächst die Hinwendung zu Grenzen als soziale Produktionen vorgestellt, die sich an Prozessen ihrer (De-)Stabilisierung festmachen lässt und in bzw. durch Praktiken manifest wird. Daran anschließend wurde eine geweitete Auffassung solcher Prozesse herausgearbeitet, die für die Vielheit der daran beteiligten Aspekte sensibilisiert und einen multiplen Zugriff auf Grenz(de)stabilisierungen einfordert. Schließlich wurde die Tendenz zu komplexeren Betrachtungen ausgeführt, die Grenz(de)stabilisierungen als Effekte von dynamischen Formationen verstehen.

\begin{tabular}{|l|l|l|l|}
\cline { 2 - 4 } \multicolumn{1}{c|}{} & Processual shift & Multiplicity shift & Complexity shift \\
\hline Grenzbegriff & Grenze als & Grenze als & Grenze als \\
& soziale Produktion & multipler Prozess & komplexe \\
& & & Formation \\
\hline $\begin{array}{l}\text { Methodische } \\
\text { Prinzipien }\end{array}$ & $\begin{array}{l}\text { Dezentrieren } \\
\text { Prozessualisieren }\end{array}$ & Multidimensionieren & Texturieren \\
& & Multilokalisieren & Relationieren \\
\hline
\end{tabular}

\section{Tabelle 1: Aktuelle analytische Trends der Grenzforschung (eigene Darstellung)}

Der Durchgang durch diese shifts hat zum einen gezeigt, dass sich die Ansätze und Begriffe der Grenzforschung kontinuierlich ausdifferenziert haben, um Grenz(de)stabilisierungen (noch) weitreichender zu erfassen und besser zu verstehen; zum anderen, dass die Idee der sozialen Gemachtheit von Grenzen von der Kategorie der sozialen Praxis angeleitet wird. Dabei weist sie eine gewisse begriffliche Variation auf bei weitgehender Verschwiegenheit darüber, was unter den analytisch bedeutsamen Grenzpraktiken, border practices, border-making processes genau zu verstehen ist. Dieses Desiderat der Grenzforschung erschwert nicht nur zu klären, was Praktiken der Grenze spezifisch macht und worauf der Forschungsfokus genau zu richten ist. Es erschwert genauso eine angemessene sozialtheoretische Fundierung vorzunehmen, die wiederum eine produktive Rückbindung von Forschungsergebnissen unterstützt. Neben vereinzelten Arbeiten, die sich explizit mit dem Praxisbegriff auseinandersetzen (vgl. z.B. CôtéBoucher et al. 2014; Wille 2014; Wille/Connor 2019), bleibt für die Grenzforschung also eine überwiegend unbestimmte Verwendung von Begriffen wie practices (vgl. Paasi 1999), border practice (Parker/Adler-Nissen 2012, S. 776), border performatives (Kaiser 2012, S. 523), Praktiken der Grenze (Wille et al. 2014, S. 10, 254ff.) oder la praxis des sujets (Auzanneau/Greco 
2018, S. 12) festzuhalten, die über das Deklarieren eines sozialkonstruktivistischen Ansatzes oft nicht hinauskommt. Ein von den beteiligten Disziplinen gemeinsam geteilter sozialtheoretischer Hintergrund ist weder durchsetzbar noch wünschenswert, im Sinne eines Ausblicks sei jedoch auf die Praxistheorien als eine mögliche Inspiration für die theoretisch-konzeptionelle Auseinandersetzung mit sozialer Praxis als Modus der Grenz(de)stabilisierung verwiesen.

$\mathrm{Zu}$ den Praxistheorien zählen sozialtheoretische Ansätze (vgl. z.B. Schatzki 2002; Reckwitz 2003; Schmidt 2012; Hillebrandt 2014; Schäfer 2016; Gherardi 2019), welche die soziale Praxis ins Zentrum stellen und eine Reihe etablierter Dichotomien überwinden (z.B. Struktur/Handlung, Regel/Ausführung, Mensch/Objekt, Mikro-/Makroeinteilungen etc.). Die sogenannte flache Ebene der sozialen Praxis - hier verstanden als Schauplatz von Grenz(de)stabilisierungen - geht mit Blick auf Individuum und Gesellschaft von einem sich wechselseitig konstituierenden und fortlaufend erneut hervorbringenden Verhältnis aus; oder in anderen Worten: Den Ort des Sozialen bildet in den Praxistheorien die sich andauernd aktualisierende Praxis. Damit sind Sozialität bzw. Grenzen als soziale Produktionen außerhalb sozialer Praxis nicht denkbar, sie werden in der Praxis beständig (re)produziert und in der Zeit transformiert. Weiter sind die Praxistheorien für die Untersuchung von Grenzen als multiple Prozesse anschlussfähig, etwa über die in Praktiken berücksichtigten Körperlichkeiten und Materialitäten. Während Körper für die (gekonnte) Aufführung von Wissen in Praktiken bedeutsam sind, nehmen sie - wie Objekte oder Artefakte - in den Praxistheorien zugleich an Praktiken teil. Körper, Objekte und Artefakte sind also konzeptionelle Bestandteile sozialer Praxis bzw. von Prozessen der Grenz(de)stabilisierung, in denen sie als soziomaterielle Allianzen oder Träger der Grenze wirksam werden können (siehe auch Bruns in diesem Band). Außerdem verstehen Praxistheorien die Praktiken mit ihren verschiedenen Bestandteilen nicht als isolierte soziale Entitäten, sondern stets eingebettet in relationale Zusammenhänge mit anderen (mitunter vergangenen) Praktiken. Diese kontextualisierende Anschauung des Sozialen, die Praxistheoretiker"innen auch als „field of practices“ (Schatzki 2001, S. 2) oder „texture of practices“ (Gherardi 2019, S. 6) bezeichnen, bietet geeignete Anschlüsse für die Untersuchung von Grenzen als komplexe Formationen. Die abschließenden Hinweise auf mögliche produktive Verschränkungen der Grenzforschung mit den Praxistheorien weisen Perspektiven und geben einen Ausblick darauf, wie die analytisch bedeutsame Kategorie der sozialen Praxis als Modus der Grenz(de)stabilisierung sozialtheoretisch rückgebunden und somit die theoretisch-konzeptionelle Debatte zwischen Grenzforschenden und über Disziplinengrenzen hinweg intensiviert werden kann.

\section{Weiterführende Literatur}

Amilhat Szary, Anne-Laure/Giraut, Frédéric (2015): Borderities: The Politics of Contemporary Mobile Borders. In: Dies. (Hrsg.): Borderities and the Politics of Contemporary Mobile Borders. Basingstoke: Palgrave, S. 1-22.

Brambilla, Chiara (2015): Exploring the Critical Potential of the Borderscapes Concept. In: Geopolitics 20, H. 1, S. 14-34.

Houtum, Henk van /Naerssen, Ton van (2002): Bordering, ordering and othering. In: Tijdschrift voor Economische en Sociale Geografie 93, H. 2, S. 125-136.

Rumford, Chris (2012): Towards a Multiperspectival Study of Border. In: Geopolitics 17, H 4, S. 887-902. Schulze Wessel, Julia (2017): Grenzfiguren - Zur politischen Theorie des Flüchtlings. Bielefeld: transcript. 


\section{Literaturverzeichnis}

Agier, Michel (2018): La Jungle de Calais. Paris: Presses Universitaires de France.

Albert, Mathias/Brock, Lothar (1996): Debordering the World of States. New Spaces in International Relations. In: New Political Science 18, H. 1, S. 69-106.

Amilhat Szary, Anne-Laure/Giraut, Frédéric (2015): Borderities: The Politics of Contemporary Mobile Borders. In: Dies. (Hrsg.): Borderities and the Politics of Contemporary Mobile Borders. Basingstoke: Palgrave, S. 1-22.

Amoore, Louise (2006): Biometric borders: governing mobilities in the war on terror. In: Political Geography 25, H. 3, S. 336-351.

Auzanneau, Michelle/Greco, Luca (2018): Introduction. Dessiner les frontières: une approche praxéologique. In: Dies. (Hrsg.): Dessiner les frontières: une approche praxéologique. Lyon: ENS Editions, S. 7-19.

Balibar, Etienne (1998): The borders of Europe. In: Pheng Cheah, Bruce Robbins (Hrsg.): Cosmopolitics. Thinking and feeling beyond the nation. Minneapolis: University of Minnesota Press, S. 216-232.

Boucher-Côté, Karine/Infantino, Federica/Salter, Mark B. (2014): Border security as practice: An agenda for research. In: Security Dialogue 45, H. 3, S. 195-208.

Brambilla, Chiara/Jones, Reece (2019): Rethinking borders, violence, and conflict: From sovereign power to borderscapes as sites of struggles. Environment and Planning D: Society and Space. DOI: $10.1177 / 0263775819856352$.

Brambilla, Chiara (2015): Exploring the Critical Potential of the Borderscapes Concept. In: Geopolitics 20, H. 1, S. 14-34.

Brambilla, Chiara/Laine, Jussi/Scott, James W./Bocchi, Gianluca (2015) (Hrsg.): Borderscaping: Imaginations and Practices of Border Making. Farnham: Ashgate.

Bürkner, Hans-Joachim (2017): Bordering, borderscapes, imaginaries: From constructivist to post-structural perspectives. In: Opiłowska, Elżbieta/Kurcz, Zbigniew/Roose, Jochen (Hrsg.): Advances in Borderlands Studies. Baden-Baden: Nomos, S. 85-107.

Casas-Cortes, Maribel/Cobarrubias, Sebastian/De Genova, Nicholas/Garelli, Glenda/Grappi, Giorgio/Heller, Charles/Hess, Sabine/Kasparek, Bernd/Mezzadra, Sandro/Neilson, Brett/Peano, Irene/Pezzani, Lorenzo/Pickles, John/Rahola, Federico/Riedner, Lisa/Scheel, Stephan/Tazzioli, Martina (2015): New Keywords: Migration and Borders. In: Cultural Studies 29, H. 1, S. 55-87.

Gerst, Dominik/Klessmann, Maria/Krämer, Hannes/Sienknecht, Mitja/Ulrich, Peter (2018): Komplexe Grenzen. Aktuelle Perspektiven der Grenzforschung. In: Berliner Debatte Initial 29, H. 1, S. 3-11.

Gherardi, Silvia (2019): Practice as a collective and knowledgeable doing. Working Paper Series 8, Collaborative Research Center 1187 Media of Cooperation.

Herzog, Lawrence A./Sohn, Christophe (2019): The co-mingling of bordering dynamics in the San Diego-Tijuana cross-border metropolis. In: Territory, Politics, Governance 7, H. 2, S. 177-199.

Hess, Sabine (2018): Border as Conflict Zone. Critical Approaches on the Border and Migration Nexus. In: Bachmann-Medick, Doris/Kugele, Jens (Hrsg.): Migration. Changing Concepts, Critical Approaches. Berlin: de Gruyter, S. 83-99.

Hillebrandt, Frank (2014): Soziologische Praxistheorien. Eine Einführung. Wiesbaden: Springer VS.

Houtum, Henk van/Kramsch, Olivier/Zierhofer, Wolfgang (2005): Prologue - B/ordering Space. In: Dies. (Hrsg.): B/ordering Space. Aldershot: Ashgate, S. 1-13.

Houtum, Henk van/Naerssen, Ton van (2002): Bordering, ordering and othering. In: Tijdschrift voor Economische en Sociale Geografie 93. H. 2, S. 125-136.

Ibrahim, Yasmin/Howarth, Anita (2018): Calais and its Border Politics: From Control to Demolition. New York: Routledge.

Jones, Reece (2019): Open Borders: In Defense of Free Movement. Athens: University of Georgia Press.

Kaiser, Robert J. (2012): Performativity and the Eventfulness of Bordering Practices. In: Wilson, Thomas M./Donnan, Hastings (Hrsg.) A Companion to Border Studies. Chicester: John Wiley \& Sons, S. 522537.

Konrad, Victor (2015): Towards a Theory of Borders in Motion. In: Journal for Borderlands Studies 30, H. 1, S. 1-17.

Konrad, Victor/Brunet-Jailly, Emmanuel (2019): Approaching borders, creating borderland spaces, and exploring the envolving borders between Canada and the United States. In: The Canadian Geographer/Le Géographe canadien 63, H. 1, S. 4-10.

Lefebvre, Henri (1991): The Production of Space. Oxford: Blackwell.

Martin, Diana/Minca, Claudio/Katz, Irit (2019): Rethinking the camp: On spatial technologies of power and resistance. Progress in Human Geography. DOI: 10.1177/0309132519856702

Newman, David (2006): Borders and Bordering: Towards an Interdisciplinary Dialogue. In: European Journal of Social Theory 9, H. 2, S. 171-186. 
Newman, David/Paasi, Anssi (1998): Fences and Neighbours in the Postmodern World: Boundary Narratives in Political Geography. In: Progress in Human Geography 22, H. 2, S. 186-207.

Oesch, Lucas (2017): The refugee camp as a space of multiple ambiguities and subjectivities. In: Political Geography 60, S. 110-120.

Paasi, Anssi (1999): Boundaries as Social Practice and Discourse: The Finnish-Russian Border. In: Regional Studies 33, H. 7, S. 669-680.

Parker, Noel/Adler-Nissen, Rebecca (2012): Picking and Choosing the „Souvereign“ Border. A Theory of Changing State Bordering Practices. In: Geopolitics 17, H. 4, S. 773-796.

Parker, Noel/Vaughan-Williams, Nick (2012): Critical Border Studies: Broadening and Deepening the "Lines in the Sand“ Agenda. In: Geopolitics 17, H. 4, S. 727-733.

Parker, Noel/Vaughan-Williams, Nick et al. (2009): Lines in the Sand? Towards an Agenda for Critical Border Studies. In: Geopolitics 14, H. 3, S. 582-587.

Pott, Andreas/Rass, Christoph/Wolff, Frank (Hrsg.) (2018): Was ist ein Migrationsregime? What Is a Migration Regime? Wiesbaden: VS Verlag für Sozialwissenschaften.

Pötzsch, Holger (2015): The Emergence of iBorder: Bordering Bodies, Networks, and Machines. In: Environment and Planning D: Society and Space 33, H. 1, S. 101-118.

Reckwitz, Andreas (2003): Grundelemente einer Theorie sozialer Praktiken. Eine sozialtheoretische Perspektive. In: Zeitschrift für Soziologie 32, H. 4, S. 282-301.

Risse, Verena (2018): Die Diversifikation von Staatsgrenzen - Anlass zu einer konzeptionellen Neubestimmung? In: Berliner Debatte Initial 29, H. 1, S. 62-72.

Rumford, Chris (2012): Towards a Multiperspectival Study of Border. In: Geopolitics 17, H. 4, S. 887902.

Rumford, Chris (2011): Seeing like a border. In: Political Geography 30, H. 2, S. 67-68.

Salter, Marc B. (2012): Theory of the /: The Suture and Critical Border Studies. In: Geopolitics, 17. H. 4, S. 734-755.

Schatzki, Theodore/Knorr Cetina, Karin/Savigny, Eike von (Hrsg.) (2001): The Practice Turn in Contemporary Theory. Routledge: London, New York.

Schatzki, Theodore (2002): The Site of the Social. University Park: Pennsylvania State University Press.

Schäfer, Hilmar (2016) (Hrsg.): Praxistheorie. Ein soziologisches Forschungsprogramm. Bielefeld: transcript.

Schmidt, Robert (2012): Soziologie der Praktiken. Konzeptionelle Studien und empirische Analysen. Frankfurt/M.: Suhrkamp.

Schulze Wessel, Julia (2017): Grenzfiguren - Zur politischen Theorie des Flüchtlings. Bielefeld: transcript.

Schulze Wessel, Julia (2016): On Border Subjects: Rethinking the Figure of the Refugee and the Undocumented Migrant. Constellations. In: International Journal of Critical and Democratic Theory 23, H. 1, S. 46-57.

Sohn, Christoph (2016): Navigating borders' multiplicity: the critical potential of assemblage. In: Area 48, H. 2, S. 183-189.

Soja, Edward W. (1989): Postmodern Geographies: The Reassertion of Space in Critical Social Theory, London: Verso.

Sontowski, Simon (2018): Speed, timing and duration: contested temporalities, techno-political controversies and the emergence of the EU's smart border. In: Journal of Ethnic and Migration Studies 44, H. 16, S. 2730-2746.

Tsianos, Vassilis/Karakayali, Serhat (2010): Transnational Migration and the Emergence of the European Border Regime: An Ethnographic Analysis. In: European Journal of Social Theory 13, H. 3, S. $373-387$.

Tyerman, Thom (2019): Everyday Borders in Calais: The Globally Intimate Injustices of Segregation. In: Geopolitics, DOI: 10.1080/14650045.2019.1631807.

Weier, Sebastian/Fellner, Astrid/Frenk, Jochen/Kazmaier, Daniel/Michely, Eva/Vatter, Christoph/Weiershausen, Romana/Wille, Christian (2018): Bordertexturen als transdisziplinärer Ansatz zur Untersuchung von Grenzen. Ein Werkstattbericht. In: Berliner Debatte Initial 29, H. 1, S. 73-83.

Wille, Christian (2014): Räume der Grenze. Eine praxistheoretische Perspektive in den kulturwissenschaftlichen Border Studies. In: Elias, Friederike/Franz, Albrecht/Murmann, Henning/Weiser, Ulrich Wilhelm (Hrsg.): Praxeologie. Beiträge zur interdisziplinären Reichweite praxistheoretischer Ansätze in den Geistes- und Sozialwissenschaften. Berlin: De Gruyter, S. 53-72.

Wille, Christian/Reckinger, Rachel/Kmec, Sonja/Hesse, Markus (2014) (Hrsg.): Räume und Identitäten in Grenzregionen. Politiken - Medien - Subjekte. Bielefeld: transcript.

Wille, Christian/Connor, Ulla (2019): Cross-border cooperation as practice formation. Perspectives for an alternative research approach. In: Beck, Joachim (Hrsg.): Transdisciplinary discourses on cross-border cooperation in Europe. Bruxelles: Peter Lang, S. 255-278. 
Wille, Christian/Fellner, Astrid M./Nossem, Eva (i.E.) (Hrsg.): Bordertextures. A Complexity Approach to Cultural Border Studies. Bielefeld: transcript,

Yuval-Davis, Nira/Wemyss, Georgie/Cassidy, Kathryn (2019): Bordering. Cambridge: Polity Press. 